\title{
A pesquisa de graduação: o caso dos TCCs da USP- Ribeirão em relação aos GTs do ENANCIB ${ }^{1}$
}

\author{
Graduate research papers from Information Science Course at USP-Ribeirão in relation to \\ research groups of ENANCIB
}

\begin{abstract}
Cláudio Marcondes de Castro Filho
Doutor em Ciência da Informação pela Escola de Comunicações e Artes, Universidade de São Paulo - USP Professor do Curso de Graduação em Ciências da Informação e da Documentação da FFCLRP/USP

E-mail: claudiomarcondes@ffclrp.usp.br
\end{abstract}

\section{Resumo}

Analisa aspectos temáticos de 84 Trabalhos de Conclusão de Curso produzidos por alunos do curso de Ciências da Informação e da Documentação da USP (Ribeirão Preto, SP) no período de 2006 a 2009; do ponto de vista metodológico, relaciona palavras-chave dos resumos dos TCCs às categorias de nove dos Grupos de Trabalho do ENANCIB; os resultados apontam maior concentração de trabalhos nas categorias da mediação e da gestão informacional, respectivamente G3 e G4 dos ENANCIBs, sem descuido de outras áreas, evidenciando a harmonia de alguns temas investigativos nos TCCs com os temas presentes na associação de pesquisa brasileira.

Palavras-chave: ENANCIB: Grupos de Trabalho. Trabalho de Conclusão de Curso. Ciência da Informação: Curso.

\begin{abstract}
Themes of 84 final undergraduate research projects from Information Science and Documentation course in São Paulo University (Ribeirão Preto, SP) ranging from 2006 to 2009 period are analysed. From methodological point of view, it relates the projects keywords to categories listed in nine of Working Groups of ENANCIBs. The results show concentration of works in the categories of mediation and information management, respectively G3 and G4 of ENANCIBs, without oversighting other research areas, showing harmony of some investigative themes of these final projects and those of Brazilian research association.
\end{abstract}

Keywords: ENANCIB: Working Groups. Undergraduate Research Projects. Information Science: Course

\section{Introdução}

A produção acadêmica gerada na universidade pode ser conhecida por diferentes veículos de comunicação, com os quais pesquisadores informam os resultados de suas pesquisas, seja em fase de conclusão ou de validar hipóteses da pesquisa. A comunicação científica utiliza alguns meios formais para a divulgação do conhecimento, e que de acordo com Gonçalves Filho e Noronha (2004, p. 60) podem ser representados pelos "artigos publicados em periódicos científicos, livros, trabalhos e comunicações em eventos da área,

\footnotetext{
${ }^{1}$ Trabalho apresentado originalmente na XI Encontro Nacional de Pesquisa em Ciência da Informação, 2010; modificado para publicação final nesta oportunidade.
}

InCID: R. Ci. Inf. e Doc., Ribeirão Preto, v. 1, n. 2, p. 48-59, jul./dez. 2010. 
resumos, abstracts, além das monografias que marcam as diferentes etapas na formação e titulação acadêmica". Essas comunicações facilitam a disseminação da informação e o acesso por parte da comunidade científica da área, como também uma maior visibilidade da produção científica de um país. Para tanto, Packer e Meneghini (2006, p.237), descrevem que a visibilidade representa a "capacidade de exposição que uma fonte ou um fluxo de informação possui de, por um lado, influenciar seu público alvo e, por outro, ser acessada em resposta a uma demanda de informação". Nesse sentido, pode-se considerar que publicações elaboradas no meio acadêmico sejam elas realizadas por docentes ou discentes são frutos de pesquisa com pretensão de visibilidade na área pretendida.

Com relação aos cursos de graduação, os discentes acumulam informações e questionamentos na área estudada, como também, participam de experiências práticas nos estágios e em projetos de pesquisa ou iniciação científica, procurando validar os seus textos, por intermédio da comunicação científica. Nesse contexto, no final do curso de graduação, o discente é obrigado a produzir um trabalho que habilite a concluir o curso. Esse trabalho, denominado Trabalho de Conclusão de Curso, ou TCC, como é mais conhecido, é requisito indispensável para obtenção de grau de bacharel. Nesse aspecto, o curso de Ciências da Informação e da Documentação, da Faculdade de Filosofia, Ciências e Letras de Ribeirão Preto (FFCLRP) da Universidade de São Paulo, insere o TCC no currículo do curso, como produção acadêmica para se obter o título de bacharel em Biblioteconomia, Ciência da Informação e Documentação.

Com a carga horária de 2790 horas, o curso de Ciências da Informação e da Documentação da FFCLRP, consta com duas disciplinas obrigatórias denominadas Desenvolvimento de Trabalho de Conclusão de Curso I e II que tem como objetivo, a realização de um trabalho de pesquisa ou de desenvolvimento na área de Ciências da Informação e Documentação, sistematizado na forma de um Trabalho de Conclusão de Curso, permitindo que o estudante se familiarize com o seu futuro ambiente de trabalho e/ou área de pesquisa.

Uma das ações do ensino de graduação é o trabalho de pesquisa e de investigação científica, e os TCCs complementam essa ação como um dispositivo para os estudantes darem continuidade para futuras pesquisas nos cursos de pós-graduação. O TCC pode também segundo Ferraz (2003, p. 5), "ser utilizado promover a carreira do profissional da informação" Nesse sentido, os TCCs contribuem para o desenvolvimento da pesquisa em determinada área 
do conhecimento, e ao mesmo tempo é pré-requisito para a obtenção do grau de bacharel. Nessa pesquisa pretende-se analisar os aspectos temáticos dos Trabalhos de Conclusão de Curso, produzidos por alunos do curso de Ciências da Informação e da Documentação, da FFCLRP da Universidade de São Paulo, de acordo com as áreas de abrangência dos Grupos de Trabalho da Associação Nacional de Pesquisa e Pós-graduação em Ciência da Informação, como também a relação entre os Trabalhos de Conclusão de Curso e a produção docente e linhas de investigação do curso analisado.

\section{Ciências da Informação e Documentação da FFCLRP/USP}

O curso de Ciências de Informação e da Documentação da Faculdade de Filosofia, Ciências e Letras, de Ribeirão Preto visa à formação de profissionais em informação e documentação, que permite atuação do formado em Ciências da Informação e Biblioteconomia. Prioriza como ênfase as áreas da Educação, Saúde e Agronegócios integradas com outros cursos da Unidade, ao quadro da formação específica e geral e tem seus fundamentos apoiados na necessidade de desenvolvimento de um campo científico e de formação relativamente novos: o das Ciências da Informação.

O curso Ciências de Informação e Documentação da Faculdade de Filosofia, Ciências e Letras, de Ribeirão Preto, da Universidade de São Paulo iniciou em 2003, ministrado em 08 semestres, no período noturno. O curso atualmente é composto por oito professores das áreas da Ciência da Informação, Educação, História, Ciências Sociais e Tecnologia. Os alunos são preparados para atuar não apenas nos ambientes tradicionais (como bibliotecas públicas, escolares, universitárias, ou de institutos de pesquisa e empresas), mas também em ampla variedade de instituições da sociedade civil como sindicatos, movimentos populares, entre outros. Os profissionais devem ser capacitados para gerir unidades de informação tanto no âmbito tradicional como no âmbito eletrônico. O curso também oferece aos discentes a opção de cursar disciplinas optativas nas áreas de educação, saúde e empresa/negócios em outras unidades da universidade. 


\section{Método: coleta de dados}

Para identificação dos assuntos dos TCCs foram utilizadas as palavras-chave dos resumos elaborados nos próprios TCCs. No período de 2006 a 2009 foram produzidos 101 TCCs, sendo o universo da pesquisa de 84 TCCs, por razões de fácil localização e por consideramos essa amostra suficiente para delinear tendências. Nesse período de 2006 a 2009 foram coletados 84 TCCs, de acordo com o Gráfico1. Vale ressaltar que o curso é oferecido apenas no período noturno com total de 40 vagas/ano e que nem todos os alunos se formam no período do curso (8 semestres), pois há casos de alunos que levam até 13 semestres para obtenção do grau, que é o tempo máximo para jubilação.

Após a verificação da quantidade de TCCs no laboratório, realizamos a análise dos TCCs extraindo as palavras-chave dos resumos e posteriormente enquadrando as áreas dos TCCs, nas categorias dos Grupos de Trabalhos do ENANCIB.

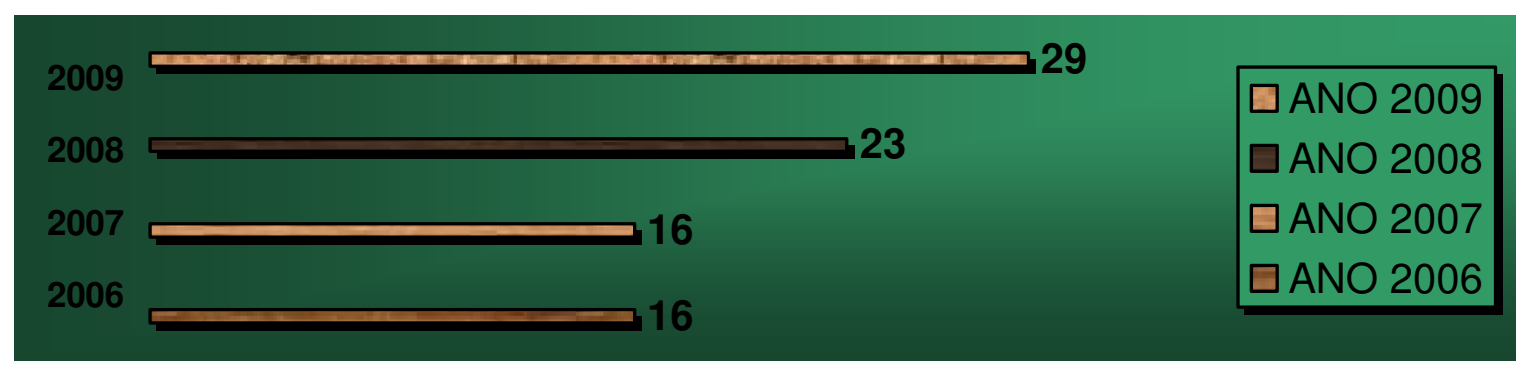

GRÁFICO 1 - Quantidade de TCCs e Ano de Defesa

\section{Resultados}

Considerando os 84 trabalhos apresentados no período de 2006 a 2009, a média anual apresentada é de 21 trabalhos, sendo que o período 2006-2007 ficou abaixo da média e o período 2008-2009 acima da média, de acordo com a Tabela 1. Esse resultado mostra que os alunos das turmas de 2006 e 2007 se atrasaram na conclusão de seus trabalhos, não completando o curso no período de 8 semestres. 
TABELA 1 - Ano de Defesa e GT`s ENANCIB

\begin{tabular}{c|c|ccccccccccc}
\hline ANO & \multicolumn{10}{c|}{ GT`s } & TOTAL & $\%$ \\
\cline { 2 - 13 } DEFESA & $\mathbf{1}$ & $\mathbf{2}$ & $\mathbf{3}$ & $\mathbf{4}$ & $\mathbf{5}$ & $\mathbf{6}$ & $\mathbf{7}$ & $\mathbf{8}$ & $\mathbf{9}$ & & \\
\hline 2006 & 0 & 3 & 1 & 5 & 1 & 5 & 0 & 1 & 0 & $\mathbf{1 6}$ & $\mathbf{1 9 , 0 5}$ \\
2007 & 0 & 3 & 3 & 3 & 1 & 1 & 0 & 1 & 4 & $\mathbf{1 6}$ & $\mathbf{1 9 , 0 5}$ \\
2008 & 1 & 5 & 3 & 3 & 0 & 3 & 0 & 5 & 3 & $\mathbf{2 3}$ & $\mathbf{2 7 , 3 8}$ \\
2009 & 1 & 3 & 9 & 5 & 1 & 3 & 2 & 5 & 0 & $\mathbf{2 9}$ & $\mathbf{3 4 , 5 2}$ \\
\hline TOTAL & 2 & 14 & 16 & 16 & 3 & 12 & 2 & 12 & 7 & $\mathbf{8 4}$ & $\mathbf{1 0 0}$ \\
\hline
\end{tabular}

\section{TABELA 2 - Distribuição dos TCCs nos GTs}

\begin{tabular}{l|cc}
\hline \multicolumn{1}{c|}{ GT'S ENANCIB } & $\begin{array}{c}\text { Quantidade } \\
\text { de } \\
\text { Trabalhos }\end{array}$ & $\%$ \\
\hline GT 1: Estudos históricos e epistemológicos da Ciência da & $\mathbf{2}$ & $\mathbf{2 , 3 8}$ \\
Informação & $\mathbf{1 4}$ & $\mathbf{1 6 , 6 6}$ \\
GT 2: Organização e Representação do Conhecimento & $\mathbf{1 6}$ & $\mathbf{1 9 , 0 5}$ \\
GT 3: Mediação, Circulação e Apropriação da Informação & $\mathbf{1 6}$ & $\mathbf{1 9 , 0 5}$ \\
GT 4: Gestão da Informação e do Conhecimento nas Organizaçães & $\mathbf{3}$ & $\mathbf{3 , 5 7}$ \\
GT 5: Política e Economia da Informação & $\mathbf{1 2}$ & $\mathbf{1 4 , 2 9}$ \\
GT 6: Informação, Educação e Trabalho & $\mathbf{2}$ & $\mathbf{2 , 3 8}$ \\
GT 7: Produção e Comunicação da Informação em CT\&I & $\mathbf{1 2}$ & $\mathbf{1 4 , 2 9}$ \\
GT 8: Informação e Tecnologia & $\mathbf{7}$ & $\mathbf{8 , 3 3}$ \\
GT 9: Museu, Patrimônio e Informação & $\mathbf{8 4}$ & $\mathbf{1 0 0}$ \\
\hline TOTAL & &
\end{tabular}

Nota-se em nossos resultados, temas com baixa concentração, como é o caso de Estudos históricos e epistemológicos e Produção e comunicação da informação em ciência e tecnologia, ambos com duas produções, temas que são mais propriamente voltados à pósgraduação, embora com sentidos diferentes, senão vejamos:

O primeiro grupo do ENANCIB, referido às preocupações epistemológicas, passou, recentemente, por análises específicas, no trabalho de Nunes e Murguia (2009), em que foi verificada maior preocupação com a epistemologia da área do que propriamente com estudos históricos; por exemplo, as categorias "estudos epistemológicos e campo científico em ciência da Informação" concentram ambos, 33\% dos termos do grupo enquanto estudos históricos apenas 5\% do total de termos. Entretanto, nossa pesquisa demonstra que o tema da epistemologia não tem sido a preocupação dos trabalhos de conclusão; isso se deve talvez à natureza dos estudos graduados, voltados que estão para aplicações práticas.

De fato, pratica-se na graduação a vigilância epistemológica mais voltada para aspectos metodológicos do próprio projeto de pesquisa discente e menos voltada para a área 
como um todo, o que parece-nos acertado, neste primeiro momento de iniciação científica. Entretanto, nossa análise aponta considerável atenção aos temas do patrimônio histórico, devido à ênfases disciplinares do curso de graduação, desde sua fundação: bibliotecas, arquivos e museus tem sido tratados em nossas disciplinas, como lugares de memória com repercussão positiva nos trabalhos de conclusão.

O tema da produção da informação em ciência e tecnologia e/ou da comunicação científica é quase originário da vertente americana da ciência da informação, tendo sido um dos temas profícuos da década de setenta, com notável ressurgimento no mundo digitalizado através da cientometria; tal tema apresenta-se pouco desenvolvido entre nós, devido à opção histórica já mencionada; nela privilegia-se mais as instituições-memória do que as áreas de conhecimento em ciência e tecnologia. Porém, destaca-se o campo médico e da saúde, com trabalhos concentrados na área de Organização e Representação do Conhecimento. Por exemplo, Terminologia do campo da saúde empregados em prontuários de pacientes: mapeamento caracterização e uso efetivo (SILVA, 2007) ou Condições e disjunções informacionais entre o CRF e o prontuário do paciente (MAGANHA, 2008).

Nossos resultados apontam certo equilíbrio entre três áreas, conforme tab. 01: Organização e Representação do Conhecimento, Mediação, Circulação e Apropriação da Informação e Gestão da Informação e do Conhecimento nas Organizações, equilíbrio que pensamos ser coerente, em princípio: a informação só circula se estiver organizada e para tal são necessárias hipóteses de organização, conforme teorizam (TÁLAMO; LARA, 2006): “[...] as linguagens de processamento e produção da informação [são] fundadas em hipóteses sobre o modo de organização de objetos integrados a conjuntos para fins de circulação do conteúdo informacional".

Nesse sentido, as hipóteses de organização levam-nos às instituições e aos contextos específicos. Assim, os trabalhos que envolvem essas três áreas em equilíbrio, incluem estudos de caso para identificação de melhores práticas de gestão para unidades de informação voltadas à inovação, a exemplo de Pasqualin (2008), bem como para identificar a importância da gestão da informação em setores da região, como é o caso do agronegócio em Ribeirão Preto, apresentado por Casanova (2008). A gestão da informação inclui também a análise dos profissionais que trabalham com informação. As organizações sucroalcooleiras da região de Ribeirão Preto são também alvo de trabalhos de final de curso neste programa, a exemplo de Moisés (2009) e Gonçalves (2009), ambos os trabalhos com a supervisão de Castro Filho, 
docente-autor com contribuição especial no tema da Gestão da Informação e do Conhecimento nas Organizações, a exemplo também de sua familiaridade com o tema das fontes de informação, em bibliotecas escolares (FERRAREZI; CASTRO FILHO, 2007).

Gestão da informação e do conhecimento nas organizações é um tema pesquisado recentemente nos trabalhos dos ENANCIB, por Silveira (2007) em que os descritores Gestão da informação, Gestão do conhecimento, Informação para decisão e Inteligência competitiva ganham proeminência na análise. O trabalho mencionado é útil pela demonstração que faz das temáticas do grupo 4, como comportamento informacional, arquitetura da informação, planejamento organizacional ou fontes de informação.

As perguntas e observações levantadas pelo autor também merecem atenção, por exemplo, estudo de usuários e necessidades de informação, são temas pertinentes ao grupo 03, mas também exercem influencia no processo de gestão da informação e do conhecimento em diversos contextos. Ressalte-se o destaque do descritor Planejamento Organizacional na mesma pesquisa de Silveira (2007) representando uma função essencial na administração das unidades de informação; ressalte-se também a dispersão dos descritores, no mesmo autor, para indicar, ora o aparecimento de termos novos na área, como é o caso de arquitetura da informação, ora a presença de termos que podem estar sendo discutidos em outros grupos de trabalho, como é o caso de estudos de usuário ou necessidades de informação, temáticas presentes na gestão da informação organizacional, mas pertinentes também ao grupo 03, referido às Mediações, circulação e apropriação da informação.

As mediações tem sido tematizadas nos trabalhos de conclusão de forma privilegiada ao enfatizar as instituições e/ou processos textuais como espaço de memória, mas também como espaço de negociação de sentidos na reflexão própria de docentes orientadores, a exemplo de Souza e Crippa (2009) ou Almeida (2008), ambos refletindo sobre o conceito de mediação nas várias teorias sociais: o primeiro no destaque à importante noção de dispositivo informacional de inspiração foucaultiana. Isso significa entender a informação ou as instituições produtoras de conhecimento como equipamentos culturais, em seus fluxos heterogêneos de coisas, processos, instrumentos e informação, onde a informação é um dos elementos do agenciamento ou da rede, tão importante quanto os prédios, as instituições ou os procedimentos.

Já a vertente desenvolvida por Almeida (2008) sinaliza a preferência por uma abordagem pós-gramsciana inspirada na sociologia de Alberto Melluci, na qual há a 
valorização do indivíduo como alguém inserido nas redes e, portanto o social será considerado por uma multiplicidade de atores, tanto individuais quanto coletivos. Exemplos da primeira abordagem são: Implantação do centro de documentação e memória do Theatro Pedro II em Ribeirão Preto: um estudo de caso (LIMA JUNIOR, 2009) ou A arte de memória no hipertexto: uma reflexão sobre o conhecimento relacional (BISOFFI, 2009). Ou ainda Os equipamentos culturais da modernidade em Ribeirão Preto: cine-teatros e cinemas (GALDINO, 2009). Na segunda abordagem estarão TCCs relacionando a questão informacional às redes sociais, a exemplo de Bombonatti (2008).

Mas, se a preferência pela concepção de dispositivo informacional serve à Crippa (2010a) para iluminar os TCCs quanto aos seus elementos heterogêneos, há ainda, no programa em análise, uma terceira vertente no tema das mediações informacionais referidas à noção de discurso: aqui a linguagem passa a ser o foco de análise privilegiado, secundarizando outros elementos do agenciamento ou da rede de atores. São exemplos desta vertente: Informação em (dis)curso: inscrição de sentido em Brazil, o filme (SILVA, 2009); Informação e discurso: sentidos em movimento (OLIVEIRA, 2009); A linguagem dos documentos administrativos da rede estadual de ensino: um estudo sobre o acesso e uso dos professores (SILVA, 2006). Constata-se assim, a riqueza de opções teóricas desenvolvida no tema das mediações informacionais.

Cabe ainda constatar o necessário crescimento dos TCCs no tema das tecnologias, como evidencia a tabela 01 e um comentário especial com relação ao tema dos museus e patrimônio ainda se faz necessário: apesar de muito recentes os grupos de trabalho 09 e 10 nos ENANCIBs, sobre museu, patrimônio, memória e colecionismo, a coleção de TCCs aqui analisada demonstra a presença dos temas históricos como o da memória e patrimônio desde 2007, com os trabalhos de Abud, Carvalho, Souza e Sato (todos TCCs concluídos em 2007) numa espécie de antecipação à criação dos referidos GTs; a temática dos museus permanece presente em 2008 nos trabalhos de conclusão de Rodrigues e Andrade, para em 2009 constatarmos temas como a representação imagética urbana no TCC de Souza (2009) ou documentos sobre a ditadura militar no TCC de Lampoglia (2009). Ressalte-se que os orientadores dos TCCs na temática referente aos grupos 09 e 10 do ENANCIB são autores reconhecidos em trabalhos recentes, seja na publicação científica, a exemplo de Crippa 
(2010a; 2010b) e Espírito Santo (2009) seja no trabalho de divulgação científica de Revistas (ESPÍRITO SANTO, 2010) ou palestras e mini-cursos na região. ${ }^{2}$

\section{Conclusão}

Um curso de graduação em Ciências da Informação e Documentação tem amplitude maior que um curso de Biblioteconomia strictu sensu. Assim, a sistematização dos TCCs aqui apresentada, coloca o referido curso de graduação, no caminho certo da prática profissional investigativa, como convém aos cursos de nível superior, com amplo leque temático e metodológico. Um curso se agencia a partir de temáticas específicas mas também a partir de interesses docentes, sempre em transformação. A composição docente nas universidades públicas se faz por concurso aberto dentro de determinadas áreas de especialização. Mas tanto os docentes, como as áreas de conhecimento percorrem caminhos, abrem sendas, bifurcam no movimento de produzir e transmitir conhecimentos e práticas profissionais. Como o nome do curso indica, a produção discente está em harmonia com boa parte dos temas investigativos hoje presentes nesta associação de pesquisa que é a ANCIB, o que indica também acerto na composição docente do programa.

\section{Referências}

ABUD, H. L. Memória e patrimônio: o processo de legitimação das instituições de memória. 2007. 79 f. Trabalho de Conclusão de Curso (Graduação) - Faculdade de Filosofia, Ciências e Letras de Ribeirão Preto, Universidade de São Paulo, 2007.

ALMEIDA, M. A. Mediações da cultura e da informação: perspectivas sociais, políticas e epistemológicas. Tendências da Pesquisa Brasileira em Ciência da Informação, v.1, n.1, 2008.

ANDRADE, M. M. G. A arte-educação como medição cultural em museus de arte. 2008. 101 f. Trabalho de Conclusão de Curso (Graduação)-Faculdade de Filosofia, Ciências e Letras de Ribeirão Preto, Universidade de São Paulo, 2008.

BISSOFI, G. C. A arte de memória no hipertexto: uma reflexão sobre o conhecimento relacional 2009. 81 f. Trabalho de Conclusão de Curso (Graduação) - Faculdade de Filosofia, Ciências e Letras de Ribeirão Preto, Universidade de São Paulo, 2009.

\footnotetext{
${ }^{2} \mathrm{O}$ grupo 10 não foi considerado para análise por ser muito recente e não conter ainda trabalhos disponibilizados para consulta.
}

InCID: R. Ci. Inf. e Doc., Ribeirão Preto, v. 1, n. 2, p. 48-59, jul./dez. 2010. 
BOMBONATTI, V. S. Análise das relações entre redes sociais e redes neurais. $2008.85 \mathrm{f}$. Trabalho de Conclusão de Curso (Graduação) - Faculdade de Filosofia, Ciências e Letras de Ribeirão Preto, Universidade de São Paulo, 2008.

CARVALHO, T. F. O patrimônio cultural urbano no município de Descalvado: propósito de um inventário. 2007. 101f. Trabalho de Conclusão de Curso (Graduação) - Faculdade de Filosofia, Ciências e Letras de Ribeirão Preto, Universidade de São Paulo, 2007.

CASANOVA, N. A importância da gestão da informação no desenvolvimento de projetos: um estudo de caso em empresas de médio e pequeno porte do setor de agronegócio no setor de Ribeirão Preto. 2008. 93 f. Trabalho de Conclusão de Curso (Graduação) - Faculdade de Filosofia, Ciências e Letras de Ribeirão Preto, Universidade de São Paulo, 2008.

CRIPPA, G. Representações iconográficas e literárias da produção de alimentos: espaços, atores e ideologias entre Antiguidade e Idade Média. Revista de Nutrição, Campinas, v. 23, n.1, p. 149-162, 2010a.

O campo da Ciência da Informação e o patrimônio cultural: reflexões iniciais para novas discussões sobre os limites da área. Encontros Bibli: Revista Eletrônica de Biblioteconomia e Ciência da Informação, Florianópolis, v.15, n.29, p. 1-23, 2010 b.

ESPÍRITO SANTO, S. M. Os corredores do café como mediação do objeto cognitivo para a Ciência da Informação. Datagramazero, Rio de Janeiro, v. 10, n.4, 2009

Traços de um colecionador público em Ribeirão Preto. Revista Museu, Rio de Janeiro, v. 2010, p. 01-04, 2010.

FERRAREZI, L.; CASTRO FILHO, C. M. Biblioteca escolar; reflexões para elaboração de um guia bibliográfico. Biblionline, João Pessoa, v. 3, n. 2, 2007

FERRAZ, M. C. C. O valor dos trabalhos de conclusão de curso para o ingresso do profissional da informação nas empresas. Perspectiva em Ciência da Informação, Belo Horizonte, v. 8, n.1, p. 88-95, jan./jun.2003.

GALDINO, R. Os equipamentos culturais da modernidade em Ribeirão Preto: cineteatros e cinemas. 2009. 93 f. Trabalho de Conclusão de Curso (Graduação) - Faculdade de Filosofia, Ciências e Letras de Ribeirão Preto, Universidade de São Paulo, 2009.

GIL, A. C. Métodos e técnicas de pesquisa social. São Paulo: Atlas, 1999.

GONÇALVES, P. As atribuições do profissional da informação no campo da gestão da informação, inteligência competitiva e capital intelectual. 2009, 97 f. Trabalho de Conclusão de Curso (Graduação) - Faculdade de Filosofia, Ciências e Letras de Ribeirão Preto, Universidade de São Paulo, 2009.

GONÇALVES FILHO, A. M.; NORONHA, D. P. Panorama temático de trabalho de conclusão de curso de Biblioteconomia. Transinformação, Campinas, v. 16, n. 1, p. 59-70, jan./abr., 2004. 
LAMPOGLIA, F. Documentos da/sobre a ditadura militar brasileira: silenciamento e esquecimento de dizeres. 2009. $121 \mathrm{f}$. Trabalho de Conclusão de Curso (Graduação) Faculdade de Filosofia, Ciências e Letras de Ribeirão Preto, Universidade de São Paulo, 2009.

LIMA JUNIOR, C. F. Implantação do centro de documentação e memória do Theatro Pedro II em Ribeirão Preto: um estudo de caso. 2009. 79 f. Trabalho de Conclusão de Curso (Graduação) - Faculdade de Filosofia, Ciências e Letras de Ribeirão Preto, Universidade de São Paulo, 2009.

MAGANHA, E. B. Condições e disjunções informacionais entre o CRF e o prontuário do paciente. 2008. 98 f. Trabalho de Conclusão de Curso (Graduação) - Faculdade de Filosofia, Ciências e Letras de Ribeirão Preto, Universidade de São Paulo, 2008.

MOISÉS, E. M. H A atuação do profissional da informação na gestão da informação e do conhecimento em organizações sucroalcooleiras. 2009. 77 f. Trabalho de Conclusão de Curso (Graduação) - Faculdade de Filosofia, Ciências e Letras de Ribeirão Preto, Universidade de São Paulo, 2009.

NUNES, L; MURGUIA MARAÑÓN, E. I. Epistemologia da ciência da informação: o GT1 em foco. In: ENCONTRO NACIONAL DA ASSOCIAÇÃO NACIONAL DE PESQUISA E POS GRADUAÇÃO EM CIÊNCIA DA INFORMAÇÃO, 10. 2009, João Pessoa. A Responsabilidade Social da Ciência da Informação. João Pessoa : Ideia, 2009. Disponível em: <http://dci2.ccsa.ufpb.br:8080/jspui/bitstream/123456789/427/1/GT\%201\%20Txt\%201\%20NUNES,\%20L.,\%20MURGUIA,\%20E.\%20Epistemologia...pdf $>$. Acesso em: 15 jun. 2010.

OLIVEIRA, D. Informação e discurso: sentidos em movimento. 2009. 83 f. Trabalho de Conclusão de Curso (Graduação) - Faculdade de Filosofia, Ciências e Letras de Ribeirão Preto, Universidade de São Paulo, 2009.

PACKER, A. L.; MENEGHINI, R. Visibilidade da produção científica. In: POBLACION, D. A.; WITTER, G. P.; SILVA, J. F. M. da. Comunicação \& produção científica: contexto, indicadores e avaliação. São Paulo: Angellara, 2006. p. 237-259.

PASQUALIN, L. O. Identificação de melhores práticas de gestão para unidades de informação voltadas à inovação: estudo de caso em dois serviços de informação. 2008. 101 f. Trabalho de Conclusão de Curso (Graduação) - Faculdade de Filosofia, Ciências e Letras de Ribeirão Preto, Universidade de São Paulo, 2008.

RODRIGUES, B. C. A ciência da informação e suas relações com arte e museu de arte. 2008. 97 f. Trabalho de Conclusão de Curso (Graduação) - Faculdade de Filosofia, Ciências e Letras de Ribeirão Preto, Universidade de São Paulo, 2008.

SANTOS, C. R. Monografias científicas: TCC - dissertação - tese. São Paulo: Avercamp, 2007.

SATO, P. A. Museu de arte de Ribeirão Preto: aspectos de relevância na aplicação social. 2007. 87 f. Trabalho de Conclusão de Curso (Graduação) - Faculdade de Filosofia, Ciências e Letras de Ribeirão Preto, Universidade de São Paulo, 2007. 
SILVA, G. M. A linguagem dos documentos administrativos da rede estadual de ensino: um estudo sobre o acesso e uso dos professores. 2006. $101 \mathrm{f}$. Trabalho de Conclusão de Curso (Graduação) - Faculdade de Filosofia, Ciências e Letras de Ribeirão Preto, Universidade de São Paulo, 2006.

SILVA, J. C. Terminologias do campo da saúde empregadas em prontuários de pacientes: mapeamento caracterizado e uso efetivo. 2007. 87 f. Trabalho de Conclusão de Curso (Graduação) - Faculdade de Filosofia, Ciências e Letras de Ribeirão Preto, 2007.

SILVA, J. R. B. Informação em (dis)curso: inscrição de sentido em Brazil, o filme. 2009. 110 f. Trabalho de Conclusão de Curso (Graduação) - Faculdade de Filosofia, Ciências e Letras de Ribeirão Preto, Universidade de São Paulo, 2009.

SILVEIRA, M. A. A. Gestão da informação e do conhecimento: análise temática dos trabalhos do VI ENANCIB. Informação e Informação, Londrina, v. 12, n. 2, jul./dez. 2007. Disponível em:

<http://www.uel.br/revistas/uel/index.php/informacao/article/view/1769/1509>. Acesso em: 17 jul. 2010.

SOUZA, W. E. R. Os monumentos de Ribeirão Preto: patrimônio, memória institucional e imaginário social. 2009. 101 f. Trabalho de Conclusão de Curso (Graduação) - Faculdade de Filosofia, Ciências e Letras de Ribeirão Preto, Universidade de São Paulo, 2009.

SOUZA, W. E. R.; CRIPPA, G. A cidade como lugar de memória: mediações para a apropriação simbólica e o protagonismo cultural. Museologia e patrimônio: Revista Eletrônica do Programa de Pós-Graduação em Museologia e Patrimônio, Rio de Janeiro, v. 2, n. 2, p. 61-72, jul/dez. 2009.

TÁLAMO, M. F. G. M.; LARA, M. L. G. O campo da lingüística documentária. Transinformação, Campinas, v. 18, n. 3, p. 203-211, set./dez. 2006.

Artigo submetido em 22 set. 2010

Artigo aceito em 29 out. 2010 